\title{
Analysis on the Reporting of Medical Events in Permanent Prostate Brachytherapy
}

\author{
Wanbao Gao ${ }^{1}$ \\ 1. Southern Advanced Medical Physics, Inc.
}

$\square$ Corresponding author: Wanbao Gao, twgao4381@hotmail.com

Disclosures can be found in Additional Information at the end of the article

\section{Abstract}

Purpose: In February 2012, the Nuclear Regulatory Commission (NRC) Advisory Committee on the Medical Use of Isotopes (ACMUI) recommended a source strength-based criterion defining a medical event (ME) in permanent brachytherapy as a procedure with greater than $20 \%$ of the sources falling outside the treatment site. A review is performed on prostate seed implants reported as MEs to regulatory agencies, aiming to analyze the nature of the events and the impact of the rulemaking on the practice of permanent prostate brachytherapy.

Methods and Materials: MEs in permanent prostate brachytherapy reported to regulatory agencies from 1999 to 2012 are reviewed and divided into three categories based on the nature of the events and the reporting methods.

Results: A total of 346 prostate seed implants were reported as MEs to NRC or agreement states from 1999 to 2012. The events consist of: (i) incorrect source strength/planning error $(n=32)$; (ii) seed/needle misplacement or excessive dose to normal tissue/organ ( $\mathrm{n}=104)$; and (iii) discrepancy in dose to target $(n=210)$. Many MEs are due to prostate volume expansions which occur after the implant procedures. Application of the recommended rules would eliminate these cases wrongly categorized as MEs.

Conclusions: Methods used for evaluating and reporting MEs in permanent prostate brachytherapy have been inconsistent over time. It is challenging to determine MEs based on dose or seed distributions without infringing on AUs' practice of medicine. The use of postimplant dosimetry for regulatory compliance purposes places the technique of permanent brachytherapy under a high level of scrutiny and has resulted in a large number of MEs after 2008 under the current dose-based standard. The practice of permanent prostate brachytherapy is, therefore, detrimentally affected. Many of these implants would not be classified as MEs when a source strength-based standard is used, as recommended recently by ACMUI.

Received 02/22/2013 Review began 02/23/2013 Published 08/01/2013

\section{(c) Copyright 2013}

Gao. This is an open access article distributed under the terms of the Creative Commons Attribution License CC-BY 3.0., which permits unrestricted use, distribution, and reproduction in any medium, provided the original author and source are credited.
Regulations should be patient outcome and impact-based by taking into account the differences in treatment and QA procedures in various radiation therapy modalities. Unless post-implant dosimetry is used strictly for QA purposes, establishment of a source strength-based ME standard for permanent brachytherapy with a $50 \%$ allowance for seeds falling outside the treatment site is warranted in order to maintain a balance in regulatory oversight of all treatment techniques. In addition, well-balanced regulations must be accompanied by rigorous institutional QA programs with the goal that high quality implants are consistently achieved and prostate cancer patients receive the full benefit from their permanent brachytherapy treatments. 
Categories: Medical Physics, Radiation Oncology, Urology

Keywords: brachytherapy, medical event, prostate, permanent brachytherapy, medical physics

\section{Introduction}

A medical event (ME) in brachytherapy is defined in 10CFR $\$ 35.3045$ as a procedure with the delivered dose differing from the prescribed dose by $20 \%$ or more. However, application of the rule in permanent prostate brachytherapy is difficult because the prescription is to a volume in which the dose distribution is inhomogeneous, and the determination of the prostate volume is associated with a number of uncertainties, depending on the timing and modality of postimplant imaging as well as the contouring technique. The difficulty is evident as shown in NRC's event reporting web page [1], where a variety of criteria have been used to report MEs in prostate implants even after January 2004, when NRC adopted $\mathrm{D}_{90}$ (the minimum dose received by $90 \%$ of the target volume) as the delivered dose to be compared with the prescribed dose.

Events reported at two facilities prompted NRC to reconsider the appropriateness and adequacy of the regulations for written directives (WDs) and MEs in permanent prostate brachytherapy. In 2003 and 2005, two prostate brachytherapy procedures performed in a Philadelphia VA Medical Center (PVAMC) were reported to NRC as possible MEs when approximately half of intended I-125 seeds fell into patients' bladders. The ME reports were subsequently retracted because, in both cases, the authorized user (AU) revised the WDs to reflect the actual number of seeds implanted in the patients after seeds being retrieved from the bladders. Therefore, they did not constitute reportable MEs. In 2003, another facility reported that 21 patients received sub-standard I-125 seed implants with $\mathrm{V}_{100}$ (the percentage target volume receiving the prescribed dose) < 80\%. In August 2008, NRC published draft rules [2] proposing to change the WD in permanent prostate seed implant from "dose-based" to "source strength-based". Under these rules, a procedure would be deemed a ME if the total source strength implanted in the treatment site is less than $80 \%$ of the total strength prescribed, or any seed is deposited beyond $3 \mathrm{~cm}$ from the treatment site, or the implant is carried out without a WD. The implementation of these rules, however, was postponed because of the then-ongoing investigation of prostate brachytherapy programs within the VA health system [3]. NRC determined it was necessary to re-evaluate the proposed rules, and requested ACMUI to provide recommendations on appropriate regulatory changes to take into account the findings from the investigations. Since then, the subject of ME definitions in permanent brachytherapy has been widely debated [4-6], primarily as to whether it should be "dose-based" or "source strengthbased", or a combination of both. American Society for Radiation Oncology (ASTRO) expressed concerns in its working group report [5], explaining that a dose-based definition of ME could lead to many properly executed prostate seed implants to be spuriously labeled as MEs due to a number of uncertainties in the post-implant analysis. For example, in NRC's inspection of PVAMC, 97 out of 116 implants were identified as MEs using the existing dose-based rules; three to 80 of them would not be classified as MEs if a source strength-based standard was applied [5, 7]. In February 2012, ACMUI proposed a source strength-based ME standard to require at least $80 \%$ of the intended source strength to be implanted in the treatment site [6]. The NRC adopted the proposal in April 2012 in developing the final rules [8].

To better understand the impact of the upcoming changes, a review was performed on all MEs in prostate seed implants notified to regulatory agencies from 1999 through 2012, aiming to analyze the nature of the events, and assess potential effects of the recent recommendations on ME identifications. A medical physicist's perspective was presented on how the rulemaking could affect the practice of permanent prostate brachytherapy.

Other than a dose error of $20 \%$ or more, there are other pathways that lead to reportable MEs (10CFR35.3045), including excessive doses to normal tissue/organ/skin, wrong patient, wrong 
treatment site, wrong isotope, wrong modality, and the use of leaking sources. It is the $20 \%$ rule that has been controversial, and this is the focus of the present study.

\section{Materials And Methods}

MEs in permanent prostate brachytherapy reported to NRC or agreement states from January 1999 to December 2012 are reviewed. They are divided into three categories based on the nature of the events and the reporting methods. Excluded from the analysis are incidents about missing shipments, lost seeds or leaking sources, aborted procedures, implants reported as possible MEs but later on retracted, or not in strict accordance with the definitions given in 10CFR35.3045.

\section{Results}

A total of 346 prostate seed implants were reported as MEs to NRC or agreement states from January 1999 to December 2012 (Figure 1), with 127 of those implants being from five medical centers within the VA health system. Many of these MEs were discovered in retrospective studies of implants performed years before they were reported. The events are divided into three categories (Figure 2):

\section{Medical Events in Permanent Prostate Brachytherapy(1999-2012)}

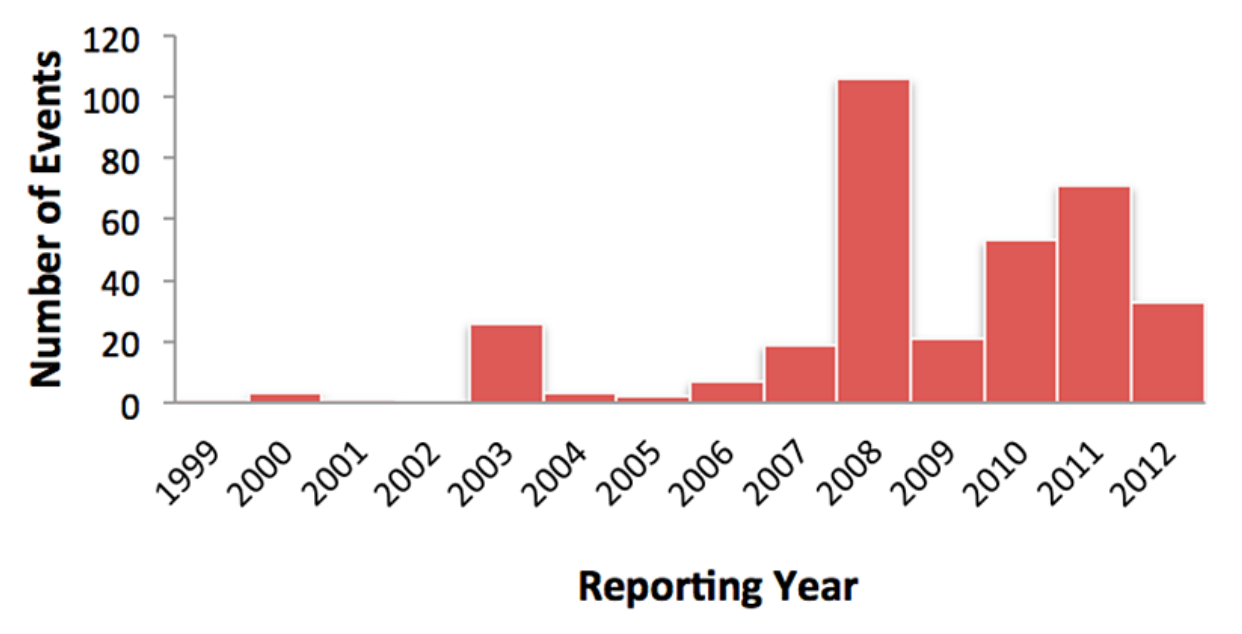

FIGURE 1: Numbers of prostate seed implants reported as MEs to regulatory agencies

Numbers of prostate seed implants reported as MEs to regulatory agencies (1999-2012). The peak at year 2008 is largely due to the investigation to PVAMC where 97 out of 116 prostate seed implants were classified as ME 


\section{Cureus}

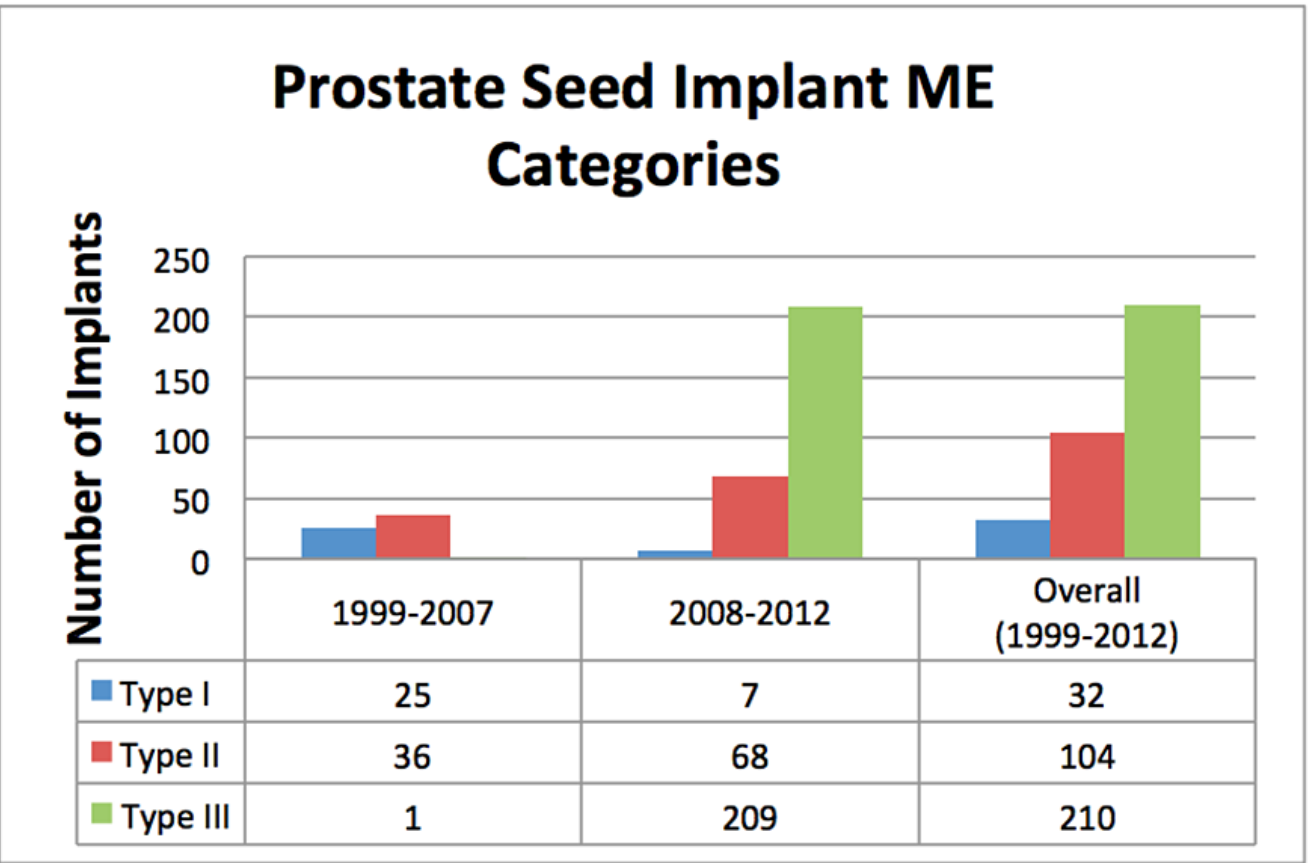

\section{FIGURE 2: Prostate seed implant ME categories based on reporting methods}

Prostate seed implant ME categories based on reporting methods: Type I - incorrect source strength/planning errors; Type II - seed/needle misplacement or excessive dose to normal tissue/organ; and Type III - discrepancy in dose to target

Type I: Incorrect source strength/planning error. There were 32 permanent seed implants reported in this category during the period reviewed with discrepancies of $\pm 20 \%$ or more between delivered and prescribed doses, ranging from -70 to $-20 \%$ and from 20 to $49 \%$. The events consist of: (a) use of incorrect source strength $(n=24)$; (b) use of incorrect dose rate constant $(n=5)$; and $(c)$ other mistakes in computer planning $(n=3)$. Examples of these MEs are listed in Table 1. 


\section{Cureus}

\section{Event Description}

$122 \mathrm{l}-125$ seeds of $0.27 \mathrm{mCi} / \mathrm{seed}$ mis-labeled as $0.414 \mathrm{mCi} / \mathrm{seed}$ due

1 to a dispensing error

2

Implant planned with $\mathrm{l}-125$ of $0.35 \mathrm{mCi} / \mathrm{seed}$, performed with 0.52 $\mathrm{mCi} / \mathrm{seed}$ due to an incorrect calculation

3

Hospital changed ordering l-125 seeds from in air kerma strength (U) to in activity $(\mathrm{mCi})$, but missing the conversion factor

$4 \quad$ Incorrect dose rate constant used in treatment plans Implant using I-125 seeds with intended dose of $107 \mathrm{~Gy}$ while the administered dose was 144 Gy

Two sets of Pd-103 seeds were ordered, the older set was used for the implant
Treatment

Error

NRC (2) or ACMUI

(6) (ME? Y/N)

$-35 \%$

$+49 \%$

Y

$+27 \%(10$

patients)

$+21 \%$ to $+36 \%$

(5 patients)

$+35 \%$

Y

$-70 \%$

Y

TABLE 1: Examples of MEs in permanent prostate brachytherapy: incorrect source strength, incorrect dose rate constant, and other planning errors

Type II: Seed/needle misplacement or excessive dose to normal tissue/organ. There were 104 procedures reported as MEs in this group, and the reporting methods were variable: (a) excessive dose to normal tissue/organ ( $n=45$, including three cases with excessive doses to urethra); (b) $V_{100}<80 \%(n=22)$; (c) wrong treatment site $(n=6)$; (d) fraction of seeds or source strength implanted inside/outside the prostate $(n=4)$; (e) needle misplacement by a distance $(1--5.5 \mathrm{~cm})$ from a reference plane $(\mathrm{n}=3)$; and (f) others (mean dose, $\mathrm{D}_{90}, \mathrm{D}_{80}$, or combinations) $(\mathrm{n}=24)$. Examples of the events are shown in Table 2. This group contains 37 out of $127 \mathrm{MEs}$ identified within the VA system. The remaining 90 MEs, reported by using $\mathrm{D}_{90}<80 \%$, are classified in the following category: 


\section{Cureus}

\begin{tabular}{|c|c|c|c|c|c|}
\hline Event & Description & $\begin{array}{l}\text { Seeds in } \\
\text { Prostate }\end{array}$ & $\begin{array}{l}\text { NRC } \\
(2) \\
(M E ? \\
\text { Y/N) }\end{array}$ & $\begin{array}{l}\text { Seeds in } \\
\text { PTV }\end{array}$ & $\begin{array}{l}\text { ACMUI } \\
\text { (6) } \\
\text { (ME? } \\
\text { Y/N) }\end{array}$ \\
\hline 1 & $\begin{array}{l}122 \mathrm{I}-125 \text { seeds were implanted } 2 \mathrm{~cm} \text { too low and missed the upper } \\
\text { portion of prostate. Adjacent tissue }(68 \mathrm{~cm} 3) \text { received the } \\
\text { prescribed dose of } 144 \mathrm{~Gy}\end{array}$ & $<80 \%$ & Y & $\begin{array}{l}\text { Margin } \\
\text { dependent }\end{array}$ & TBD(a) \\
\hline 2 & $\begin{array}{l}\text { Approximately } 5 \mathrm{l}-125 \text { seeds mistakenly placed }>1 \mathrm{~cm} \text { from the } \\
\text { prostate toward the perineum, resulting in excessive dose to } \\
\text { normal tissue }\end{array}$ & $>80 \%$ & $\mathrm{~N}$ & $>80 \%$ & $\mathrm{~N}$ \\
\hline 3 & $\begin{array}{l}93 \text { implanted } \mathrm{I}-125 \text { seeds retained the planned pattern grouping, } \\
\text { with the superior end of the seed cloud being about } 2 \mathrm{~cm} \text { from the } \\
\text { apex of the prostate. D90 }=2.24 \mathrm{~Gy} \text { and the prescribed dose }=145 \\
\text { Gy }\end{array}$ & $<80 \%$ & Y & $<80 \%$ & $\mathrm{Y}$ \\
\hline 4 & $\begin{array}{l}13 \text { out of } 63 \mathrm{I}-125 \text { seeds fell into bladder, underdose to the target } \\
\text { by more than } 20 \%\end{array}$ & $<80 \%$ & $\mathrm{Y}$ & $<80 \%$ & $\mathrm{~N}(\mathrm{~b})$ \\
\hline 5 & $\begin{array}{l}22 \text { out of } 112 \text { seeds placed outside the prostate, inferior to the } \\
\text { gland }(5.4 \mathrm{~cm}) \text { in the perineum }\end{array}$ & $>80 \%$ & $\mathrm{Y}(\mathrm{c})$ & $>80 \%$ & $\mathbf{N}$ \\
\hline
\end{tabular}

\section{TABLE 2: Examples of MEs in permanent prostate brachytherapy: seed/needle misplacement}

(a) To be determined by the PTV margin used around the prostate: $5 \mathrm{~mm}$-- likely YES; $10 \mathrm{~mm}$-- too close to project; (b) The AU shall complete the written directive to include the actual number of seeds implanted before patient is released; (c) If any seed is more than $3 \mathrm{~cm}$ away from the prostate, the implant is a ME, per NRC proposed rules in 2008 (2)

Type III: Discrepancy in dose to target. There were 210 prostate seed implants in this group during the time interval: (a) $\mathrm{D}_{90}<80 \%$ or $>120 \%(\mathrm{n}=200)$, with the absolute majority reporting underdoses; (b) 'dose to prostate' < $80 \%(\mathrm{n}=2)$; (c) $\mathrm{D}_{100}<80 \%(\mathrm{n}=1)$; (d) $\mathrm{V}_{100}<80 \%(\mathrm{n}=1)$; and (e) combinations $(n=6)$. The reports do not reveal the specific causes for the discrepancies. Examples of the events are shown in Table 3. 


\section{Cureus}

\begin{tabular}{|c|c|c|c|c|}
\hline Event & $\begin{array}{l}\text { Number of } \\
\text { Implants }\end{array}$ & Description & $\begin{array}{l}\text { Implant } \\
\text { Year(s) }\end{array}$ & $\begin{array}{l}\text { Reporting } \\
\text { Year }\end{array}$ \\
\hline 1 & 1 & V100 $=47 \%, 64 \mathrm{I}-125$ seeds implanted & 2009 & 2009 \\
\hline 2 & 6 & $\begin{array}{l}\mathrm{D} 90=75 \%, 75 \%, 77 \%, 78 \%, 121 \%, 121 \% . \mathrm{l}-125 \text { seed } \\
\text { implants }\end{array}$ & 2003-2010 & 2010 \\
\hline 3 & 1 & D100 $=25 \%, 81 \mathrm{I}-125$ seeds implanted & 2006 & 2011 \\
\hline 4 & 36 & $\mathrm{D} 90=0-80 \%, 120-162 \%$ & 2001-2009 & 2011 \\
\hline 5 & 3 & $\begin{array}{l}(\mathrm{V} 100, \mathrm{D} 90)=(70 \%, 73 \%),(63 \%, 59 \%),(65 \%, 54 \%) . \mathrm{I}-125 \\
\text { seed implants }\end{array}$ & 2009 & 2012 \\
\hline
\end{tabular}

\section{TABLE 3: Examples of MEs in permanent prostate brachytherapy: discrepancy in dose to target}

Only dose/volume parameters were provided in the original reports. Specific causes for the discrepancies were not given. It is believed that many of these MEs were actually due to prostate expansions after the implants and would not be classified as MEs under a source strength-based standard

Only dose/volume parameters were provided in the original reports. Specific causes for the discrepancies were not given. It is believed that many of these MEs were actually due to prostate expansions after the implants and would not be classified as MEs under a source strength-based standard.

\section{Discussion}

\section{Type I errors}

MEs due to uses of incorrect source strength, incorrect dose rate constant, or other errors in computer planning can be attributed to inadequate QA procedures. This type of error can be minimized by utilizing the redundancy checking mechanism [9] to verify physics parameters in the system commissioning and dose and source strength used in treatment planning and the actual implant procedure.

\section{Type II errors}

According to the original event descriptions, contributing factors for seed or needle misplacements include: misinterpretation of ultrasound image, malfunctioning of ultrasound equipment, and poor implant technique. Adequate training of all team members is essential to the success of a brachytherapy program. The following four steps can be followed to achieve high quality implants: 1 ) check and calibrate the grid coordinates in ultrasound images before the procedure; 2 ) reproduce the ultrasound probe placement as close as possible to that in the volume study (for implants using pre-plans) before starting needle insertions, and periodically verify the set-up and prostate position during the procedure, making adjustments if necessary; 3) confirm needle placements in a second view, e.g., the sagittal view, before releasing seeds; and 4) have a fluoroscopy unit available when needed.

\section{Type III errors}


Since 2008--2009 when NRC investigated prostate brachytherapy programs within the VA system, the criterion of $\mathrm{D}_{90}<80 \%$ (discrepancy in dose to target) has become the principle method in ME determinations and notifications, as displayed in Figure 2. $\mathrm{D}_{90}$ is an important parameter in correlating dose coverage with clinical outcome and often used by AUs as the quantity guiding quality improvement. However, it introduces substantial ambiguities when used for regulatory purposes in determining if a ME has occurred because the target volume computation involves large variability due to differences in the imaging modality, time elapsed between implant and imaging, and the contouring technique [5]. As an example, if a $60 \mathrm{~cm}^{3}$ sphere (approximating a prostate) is expanded by $1 \mathrm{~mm}$ in three dimensions, the enlarged volume is $68 \mathrm{~cm}^{3}$-- an increase of $13 \%$ in size. As a result, $V_{100}$ (expressed in percentage of the total prostate volume) decreases by as much as $12 \%$. Similarly, $\mathrm{D}_{90}$ decreases by a variable amount that can be larger than $12 \%$, depending on the exact dose distribution.

The high sensitivities of 3D volume calculations relative to small variations in target delineations make any dose-volume parameter (e.g., $\mathrm{D}_{90}$ or $\mathrm{V}_{100}$ ) too arbitrary to be employed in deciding whether or not a seed implant constitutes a ME. The arbitrariness is demonstrated in the re-analysis of the 97 MEs (out of 116 implants) from PVAMC showing that 80 of them were actually due to prostate volume changes after the implant procedures; they would not have been considered as MEs if a source strength-based metric was used [5, 7]. If the VA experience is any indication, it would be reasonable to expect that most of the implants in Category III and some in Category II would not be classified as MEs under a source strengthbased standard.

\section{ACMUI recommendations}

In the recent ACMUI recommendations [6]:

1) AUs are to use the total source strength and number of sources in the WD. The source strength-based criterion requires at least $80 \%$ of intended seeds to be implanted inside the treatment site (prostate, plus an AU-defined margin). The condition that no seed is to be beyond $3 \mathrm{~cm}$ from the treatment site [2] is removed;

2) For bladder or rectum, dose to any contiguous $5 \mathrm{~cm}^{3}$ shall not exceed $150 \%$ of the prescribed dose to the target. While for urethra, dose to any contiguous $5 \mathrm{~cm}^{3}$ shall not exceed $150 \%$ of the expected dose to the structure from the plan; and

3) AUs are required to complete the WD after the implant to include the radioisotope, treatment site, number of seeds, and total strength implanted. Any unusual aspect of the implant, including patient-related limitations, is to be recorded. In addition, the AU should provide a statement in this WD completion attesting that implanted sources have been placed in accordance with the planned distribution.

Under ACMUI recommended rules [6], determinations of Type I errors (Table 1) would remain unchanged. For those of Types II and III (Tables 2, 3), parameters used for evaluating and reporting are the fraction of seeds implanted in the treatment site and doses to unintended structures (bladder, rectum and urethra). Because the AU can apply a margin (e.g. $\leqslant 1 \mathrm{~cm}$ ) around the prostate or the clinical target volume (CTV) to generate the planning target volume (PTV), event $\# 2$ in Table 2 may no longer be a ME because at least $80 \%$ of the seeds are inside the PTV or treatment site. Three implants reported as MEs (Type II) for having high urethra doses $(160 \%, 161 \%$, and $152 \%$, respectively) probably will not constitute MEs, since it is unlikely that urethra volumes receiving significant doses exceeded $5 \mathrm{~cm}^{3}$. 


\section{In the author's view}

1) The recent ACMUI recommendations represent an advance in re-establishing standards to identify MEs in permanent prostate brachytherapy by eliminating the inconsistencies in ME determinations due to prostate volume changes associated with edema. Still, ambiguity exists because of the uncertainties in defining the treatment site, which is the prostate or CTV, plus an AU-defined margin that depends on the planning philosophy. The expanded volume, often referred to as PTV, is used for seed placement to ensure sufficient dose coverage to the prostate or CTV. Because the size of or the dose to the PTV has little clinical significance, the CTV-toPTV margin in post-implant analysis can be reasonably set as from 3 to $13 \mathrm{~mm}$, with a minimum $3 \mathrm{~mm}$ margin to account for uncertainties associated with CT-based contouring and interferences from seed artifacts, and

2) The practicability to set a ME standard for urethra should be further evaluated since defining dose/volume constraints to urethra would require catheterization in the post-implant imaging process, which is not a standard practice among all clinicians. Additionally, urethra dose and volume may be overestimated due to the structure expansion by the catheter.

\section{Dose-based ME definition not the only problem}

Other than the problems with using a dose-based ME definition, two additional factors contribute to the controversies in regulating permanent brachytherapy: (a) Defining MEs based on dose or seed distributions inadvertently infringe on AUs' practice of permanent implants. Because of the interactive nature of the implant procedures when AUs need to constantly make adjustments in needle positioning from computer plans due to patient-related conditions, attempting to define MEs based on dose or seed distributions would inevitably affect AUs' decision making and needle placement -- more or less, depending on how tight or how loose the regulation is designed; and (b) as a direct treatment verification, post-implant dosimetry is unmatched. Consequently, the use of post-implant analysis for regulatory compliance purposes places permanent brachytherapy under an unequalled inspection that results in higher risks in triggering MEs.

Post-implant dosimetry was adopted as the standard in permanent brachytherapy to provide physicians with the tool to evaluate implant qualities and basis for consideration of further treatment in case a less-than-desirable implant is discovered. On the other hand, it is quite certain that MEs will occur more often if these images are used for regulatory compliance purposes. It does not mean that the modality is more problematic than others, but is merely the reflection of the fact that the technique is being more scrutinized. The apparent equal criterion (20\% tolerance) is being applied to regulatory examinations that differ considerably in widths and depths, and effectively is a much higher standard to permanent brachytherapy with an "end-to-end" examination process. Therefore, an allowance greater than $20 \%$ for permanent prostate implant is merited, and can be further justified because of the inherent safety feature associated with the technique.

\section{"Radiation safety" vs. "regulatory safety or legal safety"}

Brachytherapy is characterized by delivering high dose locally to the tumor site with rapid dose falloff in the surrounding normal tissue. This normal tissue sparing effect is further enhanced in permanent procedures by utilizing low energy photon emitting isotopes. In parallel with AUs' brachytherapy practices, NRC's mission is to protect the patients' safety by detecting those treatments that cause "harm" by either overdosing normal tissue or underdosing targets, or identifying trends that could lead to patient harm [6]. A distinction should be made, however, between the "harm" that regulators intend to catch and complications or side-effects that would occur even within normal standard of radiation therapy practices. As an example, when using 
external beam IMRT (intensity-modulated radiation therapy) to treat a spherical structure (approximating a prostate) of $60 \mathrm{~cm}^{3}$ in size with a margin of $7 \mathrm{~mm}$ all around, the equivalent volume of normal tissue surrounding the prostate and within the PTV irradiated with full therapeutic dose, including portions of rectum and bladder, is about $68 \mathrm{~cm}^{3}$. At the same time, it is observed that irradiating normal tissue of this amount in a seed implant would be considered as a ME (Event \#1, Table 2). Therefore, unless post-implant dosimetry is to be used strictly for QA purposes, a more suitable source strength-based ME standard (e.g., 50\% allowance) must be established for permanent brachytherapy. Otherwise, contradicting definitions of "safety" may exist -- between two comparable normal tissue exposures, one is considered "safe" in external beam therapy, but the other is deemed "unsafe" or "harmful" in permanent seed implant. Although radiation therapy utilizing medical accelerators is beyond NRC's regulatory scope, the majority of AUs performing permanent brachytherapy do practice external beam therapy as well. If the rules are too restrictive, AUs may eventually abandon the technique and choose other modalities, such as external beam therapy, not because the alternatives are safer or clinically more advantageous but simply to avoid more frequent regulatory or legal issues.

Establishing a ME standard should ultimately be based on the clinical outcome of the modality and the impact to the modality once the standard is applied. It is essential that a balance is maintained between all treatment techniques to avoid driving clinicians away from one technique to another. Introducing a more reasonable criterion, i.e., a 50\% threshold, for permanent brachytherapy would achieve such a balance. The $50 \%$ allowance can be justified by considering the ME (Event \#1, Table 2) when the implant missed the prostate by $2 \mathrm{~cm}$, approximately half the prostate length (prostate lengths on CT or US are typically $4-5 \mathrm{~cm}$ in permanent brachytherapy). Yet, the amount of normal tissue exposed with full therapeutic dose would still be considered safe in external beam therapy. On the other hand, issues related to deficient implants like those in Table 2 must be addressed in order to take advantage of the full benefit of permanent brachytherapy by minimizing unnecessary doses to normal tissues or structures. To do so, however, other approaches have to be explored.

\section{Regulatory standard and QA standard}

In the author's opinion, the most practical and effective way to regulate permanent prostate brachytherapy without infringing on physician's practice of medicine is to establish two sets of metrics: a regulatory metric and a quality assurance metric. The regulatory metric identifies the most serious deviations as MEs, such as 1) using wrong source strength, or wrong planning by more than $\pm 20 \%$ (same as in the current regulation or ACMUI recommendations); or 2) the fraction of planned seeds falling outside of the treatment site exceeding 50\% or wrong treatment site. The quality assurance metric defines "quality control events", to be included in the institutional quality management programs. These events are less severe deviations than MEs, but exposing issues that must be resolved in order to maintain a high standard in permanent prostate brachytherapy [10]. Examples of quality control events are: 1) using wrong source strength, or wrong planning by more than $\pm 10 \%$; or 2 ) the fraction of planned seeds falling outside of the treatment site exceeding $25 \%$. In addition, urethra dose can be addressed in the QA category to allow licensees using parameters that are pertinent to various practices among AUs. Records of quality control events, as well as corrective actions, are available for review upon NRC or agreement state inspections.

At the same time, institutions should participate in radiation oncology practice accreditations, national protocols or data registry [11] for all treatment modalities, including prostate seed implant, allowing certain cases to be independently reviewed by the experts. Technical issues can then be identified and addressed through continuing education, training, and prompting ethical practice, in combination with regulatory efforts. 


\section{Conclusions}

Methods used for evaluating and reporting MEs in permanent prostate brachytherapy have been inconsistent over time. It is challenging to determine MEs based on dose or seed distributions without infringing on AUs' practice of medicine. The use of post-implant dosimetry for regulatory compliance purposes places the technique of permanent brachytherapy under a high level of scrutiny and has resulted in a large number of MEs after 2008 under the current dose-based standard. The practice of permanent prostate brachytherapy is, therefore, detrimentally affected. Many of these implants would not be classified as MEs when a source strength-based standard is used, as recommended recently by ACMUI. Regulations should be patient outcome and impact-based by taking into account the differences in treatment and QA procedures in various radiation therapy modalities. Unless post-implant dosimetry is used strictly for QA purposes, establishment of a source strength-based ME standard for permanent brachytherapy with a $50 \%$ allowance for seeds falling outside the treatment site is warranted in order to maintain a balance in regulatory oversight of all treatment techniques. In addition, well-balanced regulations must be accompanied by rigorous institutional QA programs with the goal that high quality implants are consistently achieved and prostate cancer patients receive the full benefit from their permanent brachytherapy treatments.

\section{Additional Information \\ Disclosures}

Human subjects: All authors have confirmed that this study did not involve human participants or tissue. Animal subjects: All authors have confirmed that this study did not involve animal subjects or tissue. Conflicts of interest: In compliance with the ICMJE uniform disclosure form, all authors declare the following: Payment/services info: All authors have declared that no financial support was received from any organization for the submitted work. Financial relationships: All authors have declared that they have no financial relationships at present or within the previous three years with any organizations that might have an interest in the submitted work. Other relationships: All authors have declared that there are no other relationships or activities that could appear to have influenced the submitted work.

\section{References}

1. (2013). Accessed: January 3, 2013: http://www.nrc.gov/reading-rm/doc-collections/eventstatus/event.

2. Proposed Rules. Federal Register: 73:45635 . (August 6, 2008). http://edocket.access.gpo.gov/2008/pdf/E8-18014.pdf.

3. Department of Veterans Affairs Office of Inspector General, Review of brachytherapy treatment of prostate cancer, Philadelphia, Pennsylvania and other VA medical centers, Report No. 09-02815-143. (2010). Accessed: 2012: http://www.va.gov/oig/54/reports/VAOIG09-02815-143.pdf.

4. Medical Rulemaking Workshops for Discussion of Topics Related to Medical Part 35 Regulations (New York, NY). (2011). Accessed: 2012: http://www.blsmeetings.net/NRCMedicalRulemakingWorkshop/presentations.cfm.

5. Nag S, Demanes DJ, Hagan M, et al: Definition of medical event is to be based on the total source strength for evaluation of permanent prostate brachytherapy: A report from the American Society for Radiation Oncology. Practical Radiat Oncol. 2011, 1:218-223.

6. Advisory Committee on the Medical Use of Isotopes (ACMUI) Permanent Implant Brachytherapy Final Report (modified) . (February 2012). http://pbadupws.nrc.gov/docs/ML1201/ML12019A196.pdf.

7. Hagan M, Saleh H, Moore M, et al: Regulatory evaluation of prostate volume implants: Pitfalls of retrospective assessment. Brachytherapy. 2012, 10:385-394.

8. Advisory Committee on the Medical Use of Isotopes (ACMUI) meeting . (April 16-17, 2012). 


\section{Cureus}

http://pbadupws.nrc.gov/docs/ML1210/ML12101A246.pdf.

9. Kutcher G, Coia L, Gillin M, et al: Comprehensive QA for radiation oncology: Report of AAPM Radiation Therapy Committee Task Group 40. Med. Phys.. 1994, 21:581-618.

10. Davis B, Horwitz E, Lee R, et al: American Brachytherapy Society consensus guidelines for transrectal ultrasound-guided permanent prostate brachytherapy. Brachytherapy. 2012, 11:619.

11. Palta J, Efstathiou J, Bekelman J, et al: Developing a national radiation oncology registry: From acorns to oaks. Practical Radiat Oncol. 2012, 2:10-17. 